\title{
Odd-frequency superconductivity and Meissner effect in the doped topological insulator $\mathrm{Bi}_{2} \mathrm{Se}_{3}$
}

\author{
Johann Schmidt, Fariborz Parhizgar, and Annica M. Black-Schaffer \\ Department of Physics and Astronomy, Uppsala University, Box 516, SE-751 20 Uppsala, Sweden
}

(Received 6 September 2019; accepted 6 May 2020; published 28 May 2020)

\begin{abstract}
Doped $\mathrm{Bi}_{2} \mathrm{Se}_{3}$ is proposed to be a nematic superconductor originating from unusual interorbital pairing. We calculate all induced superconducting pair correlations in $\mathrm{Bi}_{2} \mathrm{Se}_{3}$ and discover that intraorbital odd-frequency pairing clearly dominates over a significant range of frequencies. Moreover, we explore the contributions of even- and odd-frequency pairing to the Meissner effect, including separating intra- and interband processes in the response function. Contrary to expectations, and due to interband contributions, we find a diamagnetic Meissner effect from the odd-frequency pairing that stabilizes the superconducting order.
\end{abstract}

DOI: 10.1103/PhysRevB.101.180512

Superconductivity in $\mathrm{Bi}_{2} \mathrm{Se}_{3}$ has been a focal point of experimental and theoretical research in the past few years. $\mathrm{Bi}_{2} \mathrm{Se}_{3}$ had already gained prominence as a strong topological insulator $[1,2]$, when it was discovered that electron doping by intercalation of $\mathrm{Cu}$ (and later $\mathrm{Nb}$ and $\mathrm{Sr}$ ) leads to the appearance of superconductivity [3]. Due to the strong spinorbit coupling, the pairing was already early on proposed to be of odd parity, making doped $\mathrm{Bi}_{2} \mathrm{Se}_{3}$ an intrinsic topological superconductor [4]. More recently, a series of experiments have additionally discovered a surprising breaking of the rotation symmetry of $\mathrm{Bi}_{2} \mathrm{Se}_{3}$ in the superconducting state [5-10]. This so-called nematic superconducting state has been found to only be consistent with an exotic interorbital order parameter, meaning the Cooper pairs consist of two electrons from different orbitals in the $\mathrm{Bi}_{2} \mathrm{Se}_{3}$ low-energy structure [11], in contrast to more standard intraorbital pairing.

In the presence of an additional electronic degree of freedom, such as the orbital index in $\mathrm{Bi}_{2} \mathrm{Se}_{3}$, the symmetry classification of superconducting pairing is extended [12,13]. In particular, in the presence of spin, spatial parity, orbital (or band, layer, valley, etc.), and time/frequency quantum numbers, a total eight classes are possible, of which four are odd in frequency $[12,13]$. Odd-frequency superconductivity has the electrons paired at different times, with an odd relative time difference. It was originally envisaged as an order parameter for ${ }^{3} \mathrm{He}$ [14] and has played a major role in the understanding of proximity-induced superconductivity in heterostructures [15-22]. More recently, bulk systems in the presence of interorbital terms in the Hamiltonian have also been shown to often host odd-frequency pair correlations [12,23-27].

As odd-frequency pairing joins electrons at different times, direct probes of this intrinsically dynamical state have proven

Published by the American Physical Society under the terms of the Creative Commons Attribution 4.0 International license. Further distribution of this work must maintain attribution to the author(s) and the published article's title, journal citation, and DOI. Funded by Bibsam. to be challenging. Nevertheless, there still exist directly measurable consequences, such as finite Josephson currents through half metals and Weyl semimetals, entirely carried by odd-frequency pairs [28-31]. In addition, the presence of odd-frequency correlations has been connected indirectly to, for example, the Kerr effect [26,27]. Another direct physical probe of odd-frequency pairing has traditionally been the Meissner effect. In contrast to the usual diamagnetic Meissner response of even-frequency pairing, which expels an external magnetic field from the superconductor, quasiclassical approaches have predicted a telltale paramagnetic Meissner effect for the proximity-induced odd-frequency pairing in heterostructures [22,32-34], which was recently also experimentally observed in superconductor-ferromagnet junctions [35]. A paramagnetic response for odd-frequency pair correlations has also been calculated for generic multiband Hamiltonians [36]. Because a paramagnetic Meissner effect means the superconductor attracts instead of repels magnetic fields, the superconducting state should become unstable [37]. While an odd-frequency order parameter has been shown to possibly be thermodynamically stable [38,39], the paramagnetic response from odd-frequency pair correlations would prove detrimental to superconductivity in multiorbital systems. It would therefore be very important to discover odd-frequency pairing with a diamagnetic Meissner response.

In this Rapid Communication, we calculate all pair correlations induced in the interorbital nematic superconductor $\mathrm{Bi}_{2} \mathrm{Se}_{3}$ and discover prominent intraorbital pairing terms, which are odd in frequency and clearly dominate the evenfrequency pairing over a wide range of frequencies. Moreover, we calculate the Meissner response of both the evenand odd-frequency pair correlations and analyze inter- and intraband processes separately. Surprisingly, we obtain a diamagnetic Meissner effect for the odd-frequency components. This is both paramount for the stability of the dominant oddfrequency pairing in the superconducting phase of $\mathrm{Bi}_{2} \mathrm{Se}_{3}$ and opens a road for designing other stable odd-frequency superconductors. Finally, we also find a clear twofold rotational symmetry in the Meissner response signaling the nematic state. 
Model. The low-energy physics in the normal state of $\mathrm{Bi}_{2} \mathrm{Se}_{3}$ is well captured by a linear momentum model with two orbitals $[4,40]$,

$$
\hat{\mathcal{H}}_{0}=m \sigma_{x}+v\left(k_{x} s_{y}-k_{y} s_{x}\right) \otimes \sigma_{z}+v_{z} k_{z} \sigma_{y}-\mu,
$$

where $\sigma_{i}$ and $s_{i}$ indicate the Pauli matrices in orbital and spin spaces, respectively, $v$ and $v_{z}$ are the Fermi velocities of the electrons in and out of plane, $m$ hybridizes the different orbitals, and $\mu$ is the chemical potential. We also set $\hbar=1$. The eigenvalues of $\hat{\mathcal{H}}_{0}$ are given by $\epsilon_{ \pm}^{0}=$ $\pm \sqrt{m^{2}+v^{2}\left(k_{x}^{2}+k_{y}^{2}\right)+v_{z}^{2} k_{z}^{2}}-\mu$, forming a gapped threedimensional (3D) Dirac dispersion, with the twofold degenerate valence and conduction bands separated by a $2 m$ energy gap.

We introduce superconductivity through the pairing matrix $\hat{\Delta}$ and write the full Hamiltonian in Nambu space as $\check{H}=$ $\check{H}_{0}+\check{\Delta}$, where $\check{H}_{0}=\left(\begin{array}{cc}\hat{\mathcal{H}}_{0}(\mathbf{k}) & 0 \\ 0 & -\hat{\mathcal{H}}_{0}^{*}(-\mathbf{k})\end{array}\right)$, and $\check{\Delta}=\left(\begin{array}{cc}0 & \hat{\Delta} \\ \hat{\Delta}^{\dagger} & 0\end{array}\right)$, where we use $\hat{\cdots}(\therefore)$ to signal $4 \times 4(8 \times 8)$ matrices. Out of the four possible $\mathbf{k}$-independent pairing symmetries identified for doped $\mathrm{Bi}_{2} \mathrm{Se}_{3}$ [4], the experimental discovery of nematic superconductivity $[5,6]$ singles out an unconventional frequency-independent interorbital spin-triplet order parameter. Specifically, this state transforms according to the 2D $E_{u}$ irreducible representation of the $D_{3}$ point group of $\mathrm{Bi}_{2} \mathrm{Se}_{3}$ and has the form $\left(\hat{\Delta}_{x}, \hat{\Delta}_{y}\right)=\Delta\left(s_{0} \otimes i \sigma_{y}, s_{z} \otimes i \sigma_{y}\right)$ [11]. Below the transition temperature $T_{c}$, the order parameter can form any linear combination, parametrized by $\hat{\Delta}=A_{x} \hat{\Delta}_{x}+$ $A_{y} \hat{\Delta}_{y}$ with coefficients $A_{x, y}$. The nematic state is formed by $\left(A_{x}, A_{y}\right)=[\cos (\theta), \sin (\theta)]$, where $\theta$ corresponds to the inplane angle of the nematic director. With complex coefficients $\left(A_{x}, A_{y}\right)=\frac{1}{\sqrt{2}}(1, i)$ the order parameter instead describes a chiral superconductor.

For all numerical evaluations we set $\Delta=0.3 \mathrm{meV}$, which is similar to values measured in scanning tunneling experiments [41]. The other parameters are obtained from comparison to density functional theory (DFT) calculations as $m=-0.28 \mathrm{eV}, v=0.434 \mathrm{eV}$, and $v_{z}=-0.248 \mathrm{eV}$ [40]. Superconductivity in $\mathrm{Bi}_{2} \mathrm{Se}_{3}$ is observed for electron doping [42] and we therefore study $0.27 \mathrm{eV}<\mu<0.4 \mathrm{eV}$, ranging from the Fermi level close to the bottom of the bulk conduction band to the doped metallic regime.

Odd-frequency pairing. We first perform a complete classification of the superconducting pair correlations by studying the anomalous Green's function $\hat{\mathcal{F}}$, which is directly obtained from the Matsubara Green's function

$$
\check{G}(i \omega)=(i \omega-\check{H})^{-1}=\left(\begin{array}{cc}
\hat{\mathcal{G}} & \hat{\mathcal{F}} \\
\hat{\overline{\mathcal{F}}} & \hat{\mathcal{G}}
\end{array}\right) .
$$

Here, $\hat{\mathcal{G}}$ and $\hat{\overline{\mathcal{G}}}$ are the normal Green's functions in particle and hole spaces, respectively. To obtain compact analytical expressions for all types of pair correlations, we treat, in a first step, the superconducting order parameter $\Delta$ as a small quantity relative to the other energy scales and expand the Green's function to first order in $\Delta$. The anomalous part then reduces to $\hat{\mathcal{F}}^{(1)}=\hat{\mathcal{G}}_{0} \hat{\Delta} \hat{\overline{\mathcal{G}}}_{0}$, where $\hat{\mathcal{G}}_{0}$ is the Green's functions of the bare Hamiltonian $\hat{\mathcal{H}}_{0}$.
TABLE I. Symmetry classification of the pair correlations in $\hat{\mathcal{F}}^{(1)}$ for generic $\left(A_{x}, A_{y}\right)$ according to their spin, parity, orbital, and frequency symmetries. The bold term has the same symmetries as the order parameter, while the italic (first row) highlights the $s$-wave intraorbital odd-frequency pairing. Here, $A_{ \pm}=A_{x} \pm i A_{y}, A$. $k=A_{x} k_{x}+A_{y} k_{y}, A \times k=A_{x} k_{y}-A_{y} k_{x}$, and $D=\prod_{i}\left[(i \omega)^{2}-\left(\epsilon_{i}^{0}\right)^{2}\right]$ is the common denominator with $\epsilon_{i}^{0}$ the eigenvalues of $\hat{\mathcal{H}}_{0}$.

\begin{tabular}{lcccc}
\hline \hline Pairing $\left(\mathcal{F}^{(1)} * D\right)$ & Spin & Parity & Orbital & Freq. \\
\hline $2 i A_{ \pm} m \omega \Delta$ & $\uparrow \uparrow, \downarrow \downarrow$ & $s$ & Intra & Odd \\
$2 i(A \times k) v \omega \Delta$ & $\uparrow \downarrow-\downarrow \uparrow$ & $p_{x, y}$ & Even-inter & Odd \\
$2 A_{ \pm} k_{z} v_{z} \mu \Delta$ & $\uparrow \uparrow, \downarrow \downarrow$ & $p_{z}$ & Intra & Even \\
$2(A \cdot k) v m \Delta$ & $\uparrow \downarrow+\downarrow \uparrow$ & $p_{x, y}$ & Intra & Even \\
$2(A \times k) v k_{z} v_{z} \Delta$ & $\uparrow \downarrow-\downarrow \uparrow$ & $d$ & Intra & Even \\
$\mathbf{i A}_{ \pm}\left(\mathbf{m}^{2}-\mu^{2}+\omega^{2}\right) \Delta$ & $\uparrow \uparrow, \downarrow \downarrow$ & $\mathbf{S}$ & Odd-inter & Even \\
$i A_{ \pm} k_{z}^{2} v_{z}^{2} \Delta$ & $\uparrow \uparrow, \downarrow \downarrow$ & $d$ & Odd-inter & Even \\
$2 A_{ \pm} k_{z} v_{z} \Delta$ & $\uparrow \uparrow, \downarrow \downarrow$ & $p_{z}$ & Even-inter & Even \\
$2(A \cdot k) v \mu \Delta$ & $\uparrow \downarrow+\downarrow \uparrow$ & $p_{x, y}$ & Even-inter & Even \\
\hline \hline
\end{tabular}

An overview of all pairing terms in doped $\mathrm{Bi}_{2} \mathrm{Se}_{3}$ as elucidated by $\hat{\mathcal{F}}^{(1)}$ for any choice of $\left(A_{x}, A_{y}\right)$ and with a classification according to their spin, parity, orbital, and frequency symmetries is shown in Table I [see Supplemental Material (SM) for the full anomalous Green's function [43], fully consistent with Table I]. One of the eight different terms constitutes spin-triplet, $s$-wave, interorbital, even-frequency pairing, i.e., the same symmetry as the order parameter (bold in Table I). Apart from this, there is only one other $s$-wave term, i.e., $k$ independent: a spin-triplet, intraorbital, oddfrequency pairing (italic, first row). Thus, even though the superconducting order parameter has unconventional interorbital symmetry, there exists odd-frequency spin-triplet, but otherwise conventional intraorbital $s$-wave pairing, explicitly induced by the hybridization between orbitals $m$. We also obtain another odd-frequency term, with spin-singlet, $p$-wave, even interorbital symmetry. Both of these odd-frequency (and all even-frequency) pair correlations are proportional to $A_{x}$ and $A_{y}$ in such a way, that they are present in both the nematic and chiral superconducting phases.

We find that the induced odd-frequency pair amplitudes exceed the even-frequency amplitudes with the same spatial parity for a wide range of frequencies. Comparing the even- and odd-frequency $s$-wave pair amplitudes in $\hat{\mathcal{F}}^{(1)}$, the odd-frequency pairing dominates for $m+\mu<|\omega|<-m+$ $\mu$, when the system is doped in the bulk regime, i.e., $\mu>$ $|m|$. The odd-frequency $p$-wave pairing becomes larger than the two even-frequency in-plane $p$-wave pair amplitudes for $|\omega|>m$, and $|\omega|>\mu$, respectively. These analytical findings are corroborated by a numerical analysis to infinite order in $\Delta$. We compare pair amplitudes as a function of frequency by integrating the absolute value $|\hat{\mathcal{F}}(i \omega)|$ over $\mathbf{k}$ making the usual replacement $i \omega \rightarrow \omega \pm i 0^{+}$. To study $p$-wave symmetries we multiply by the corresponding form factors before the integration. The window of frequencies in which the odd-frequency pairing dominates is clearly visible in Fig. 1 and agrees well with the analytical prediction from first-order perturbation theory (shaded areas), which also further strengthens the confidence in our perturbative analysis. These results clearly demonstrate that doped $\mathrm{Bi}_{2} \mathrm{Se}_{3}$ has dominating odd-frequency 
(a)

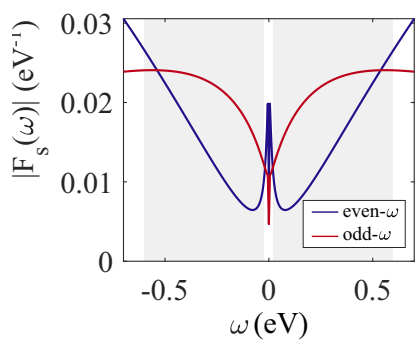

(b)

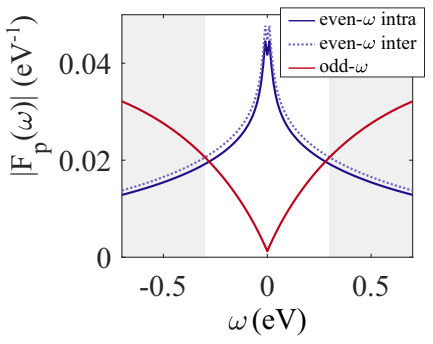

FIG. 1. Comparison between the absolute value $|\hat{\mathcal{F}}(\omega)|$ integrated over $\mathbf{k}$ for odd- and even-frequency pairing in the nematic state as a function of frequency for (a) $s$-wave and (b) in-plane $p$-wave pair amplitudes. Shaded areas mark the frequency windows at which the odd-frequency exceeds the even-frequency amplitudes obtained analytically in first-order perturbation theory. Here, the nematic angle is $\pi / 6$.

superconducting pair correlations. Taking this odd-frequency pairing into account is important for any physical property sensitive to the symmetry of the superconducting pairing, such as the Josephson effect.

Meissner effect. Having established the presence of dominant odd-frequency pairing in doped $\mathrm{Bi}_{2} \mathrm{Se}_{3}$, we turn to its influence on the Meissner effect. In contrast to the traditional diamagnetic Meissner effect of even-frequency superconductivity, odd-frequency pairing has traditionally been assumed to manifest itself in a paramagnetic Meissner response, which destabilizes the superconducting order [22,32-36].

The Meissner effect is the response of a superconductor to an external magnetic field. Within linear response theory the current response $j_{\mu}\left(\mathbf{q}, \omega_{e}\right)$ to an external vector potential $A_{\mu}\left(\mathbf{q}, \omega_{e}\right)$ is governed by the current-current response function through $j_{\mu}\left(\mathbf{q}, \omega_{e}\right)=-K_{\mu \nu}\left(\mathbf{q}, \omega_{e}\right) A_{\nu}\left(\mathbf{q}, \omega_{e}\right)$. Here, $\mathbf{q}$ and $\omega_{e}$ are the wave vector and angular frequency of the external vector potential, and $\mu$ and $v$ spatial indices $x, y, z$. The Meissner response is obtained in the limit of a static, uniform magnetic field $\omega_{e} \rightarrow 0, q \rightarrow 0$ (in that order) [47,48].

We introduce the vector potential in our Hamiltonian Eq. (1) by replacing $\mathbf{k} \rightarrow \mathbf{k}-\mathbf{A}$ (setting $e=1$ ) and calculate the current density operators by taking a variational derivative with respect to $A_{\mu}$ [49]. Usually, the current density consists of a paramagnetic $\left(j^{P} \propto A_{\mu}^{0}\right)$ and a diamagnetic part $\left(j^{D} \propto A_{\mu}\right)$. However, due to the linear spectrum of the Hamiltonian $\hat{\mathcal{H}}_{0}$, the diamagnetic current vanishes and the current-current response function reduces to $K_{\mu \nu}\left(\mathbf{q}, \omega_{e}\right)=$ $\left\langle j_{\mu}^{P}\left(q, \omega_{e}\right) j_{v}^{P}\left(-q, \omega_{e}\right)\right\rangle$ [50] (see SM for the full derivation [43]). We express this expectation value to infinite order in $\Delta$ with the help of the Green's function as

$$
\begin{aligned}
K_{\mu \nu} & =\lim _{\mathbf{q} \rightarrow 0} \lim _{\omega_{e} \rightarrow 0} K_{\mu \nu}\left(\mathbf{q}, \omega_{e}\right)=T \sum_{\mathbf{k}, i \omega} \operatorname{Tr}_{e}\left[\check{G}_{j}^{P} \breve{G}_{\mu} \breve{j}_{\nu}^{P}\right] \\
& =T \sum_{\mathbf{k}, i \omega} \operatorname{Tr}\left[\hat{\mathcal{G}} \hat{j}_{\mu}^{P} \hat{\mathcal{G}} \hat{j}_{v}^{P}+\hat{\mathcal{F}} \hat{\bar{j}}_{\mu}^{P} \hat{\overline{\mathcal{F}}} \hat{j}_{v}^{P}\right],
\end{aligned}
$$

where $\operatorname{Tr}_{e}$ is a trace over only the particle part of the matrix, $T$ is the temperature, and $\hat{j}_{\mu}^{P}=-\partial \hat{\mathcal{H}}_{0}(\mathbf{k}-\mathbf{A}) / \partial A_{\mu}$ and $\hat{\bar{j}}_{\mu}^{P}=-\hat{\mathbf{j}}_{\mu}^{P *}$ are the paramagnetic current operators in particle

and hole space, respectively. We have also suppressed the momentum and frequency dependence for legibility.

Due to the linear dispersion of the Hamiltonian with an infinite bandwidth, the integration over the first term of Eq. (3) diverges. Furthermore, it contributes an unphysical Meissner response in the limit $\Delta \rightarrow 0$, but which would be canceled by the diamagnetic current from higher-order terms of a more material-specific Hamiltonian [50]. However, because the even- and odd-frequency pair correlations are naturally fully contained in the anomalous Green's function $\hat{\mathcal{F}}=\hat{\mathcal{F}}^{e}+\hat{\mathcal{F}}^{o}$, we can work out their complete influence on the Meissner response by focusing solely on the last term in Eq. (3) [36]. Additionally, in the limit of small $\Delta$, applicable for $\mathrm{Bi}_{2} \mathrm{Se}_{3}$, the last term in Eq. (3), even captures the full Meissner effect, because the diverging first term is then always canceled by the normal-state contribution of $K_{\mu \nu}^{(0)}=\operatorname{Tr}\left[\hat{\mathcal{G}}^{0} \hat{j}_{\mu}^{P} \hat{\mathcal{G}}^{0} \hat{j}_{\nu}^{P}\right]$ [50], as we also numerically confirm (see SM [43]).

Focusing on the contribution of the second term in Eq. (3), $K_{\mu \nu}^{(S)}$, we find a sum of even- and odd-frequency contributions $K_{\mu \nu}^{(S)}=K_{\mu \nu}^{e}+K_{\mu \nu}^{o}=T \sum_{\mathbf{k}, i \omega} \operatorname{Tr}\left[\hat{\mathcal{F}}^{e} \hat{\bar{j}}_{\mu} \hat{\mathcal{F}}^{e} \hat{j}_{\nu}+\hat{\mathcal{F}}^{o} \hat{\bar{j}}_{\mu} \hat{\overline{\mathcal{F}}}^{o} \hat{j}_{\nu}\right]$ since the terms $\operatorname{Tr}\left[\hat{\mathcal{F}}^{e} \hat{\bar{j}}_{\mu} \hat{\overline{\mathcal{F}}}^{o} \hat{j}_{\nu}\right], \operatorname{Tr}\left[\hat{\mathcal{F}}^{o} \hat{\bar{j}}_{\mu} \hat{\overline{\mathcal{F}}}^{e} \hat{j}_{v}\right]$ vanish. The sign of $K_{\mu \nu}^{(S)}$ determines whether the Meissner response is diamagnetic $\left(K_{\mu \nu}^{(S)}>0\right)$ or paramagnetic $\left(K_{\mu \nu}^{(S)}<0\right)$ [36]. To further simplify the results, we use the bands obtained from diagonalizing $\breve{H}$ to split the response function $K_{\mu \nu}^{(S)}$ into intraand interband contributions. The intraband contributions are dominated by quasiparticles excited just above the superconducting gap, while the interband contribution, on the other hand, becomes enhanced when two bands approach each other in the vicinity of, but not necessarily at, the Fermi surface. After carrying out the Matsubara summation analytically, we integrate numerically over $\mathbf{k}$ at $T=2 \mathrm{~K}$ (see SM [43] for further details).

The resulting even- and odd-frequency contributions to the Meissner effect in the nematic state are presented in Figs. 2(a) and 2(b) as a function of chemical potential. For the even-frequency contribution we find a standard diamagnetic Meissner response, which is also the dominant contribution to the total Meissner response, especially for $\mu \gg m$. For the odd-frequency contribution we find that it also contributes a diamagnetic and not paramagnetic Meissner response, for almost all field directions and parameters. Considering that odd-frequency pairing has been widely considered to yield a paramagnetic response [22,32-36], this is a surprising finding, which we can understand by separately studying the intraand interband processes. In Figs. 2(c) and 2(d) we display separately the intra- and interband contributions to the $K_{x x}^{(S)}$ component at doping levels around the onset of the conduction band. For intraband processes, the even-frequency pairing gives rise to a diamagnetic and the odd-frequency pairing to a paramagnetic Meissner response, as expected. The interband processes, however, contribute with the opposite sign. The total response can then be para- or diamagnetic depending on the balance between intra- and interband processes. The even-frequency pairing yields a dominating diamagnetic Meissner response, because the intraband contribution is much larger than the interband one for $\mu>|m|$, as clearly seen in Fig. 2(c). For the odd-frequency pairing in doped $\mathrm{Bi}_{2} \mathrm{Se}_{3}$ we find intra- and interband processes of 
(a)

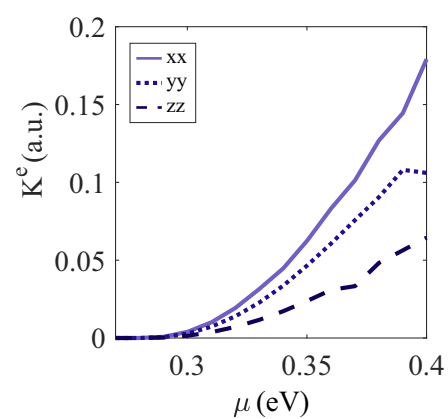

(c)

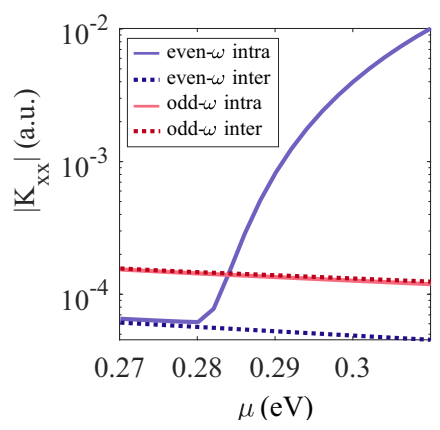

(d)

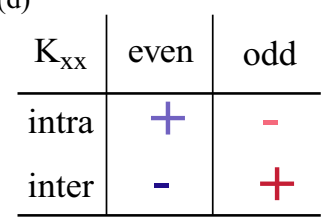

FIG. 2. Superconducting contributions to the Meissner effect for the nematic state as a function of chemical potential. (a) and (b) $K_{x x}^{(S)}, K_{y y}^{(S)}$, and $K_{z z}^{(S)}$ responses for even- and odd-frequency contributions, respectively. (c) Intra- and interband parts of the $K_{x x}^{(S)}$ Meissner response for the even- and odd-frequency contributions around the onset of the conduction band. (d) Summary of signs ( + for diamagnetic, - for paramagnetic) for the even- and odd-frequency, intra- and interband contributions to $K_{x x}^{(S)}$ displayed in (c). Here, the nematic angle is $\pi / 6$.

the same order of magnitude and also notably larger than the corresponding even-frequency processes below the onset of the conduction band. For the realistic parameters used in Fig. 2(c), the odd-frequency contributions can be strongly diamagnetic, when the interband processes become dominant, but also slightly paramagnetic such as for the $K_{y y}^{(S)}$ component in Fig. 2(b). As a consequence, the dominating odd-frequency pairing in doped $\mathrm{Bi}_{2} \mathrm{Se}_{3}$ actually contributes to a stabilizing diamagnetic Meissner effect. This is at odds with the previous notion of a paramagnetic and thus destabilizing Meissner response from odd-frequency pairing and also opens the possibility of finding other stable multiorbital odd-frequency superconductors.

Nematicity. Finally, we discuss the nematicity of the Meissner response, which is already apparent in Fig. 2, where $K_{x x}^{(S)}>K_{y y}^{(S)}>K_{z z}^{(S)}$. A different response in the $z$ direction is expected from the different Fermi velocities, $|v|>\left|v_{z}\right|$. The in-plane Fermi surface is, however, isotropic, such that the observed nematicity in the in-plane Meissner response, with $K_{x x}^{(S)}$ almost twice as large as $K_{y y}^{(S)}$ for $\theta=\pi / 6$ in Fig. 2, constitutes a clear imprint of nematic superconductivity. The nematicity also appears in the response for different nematic angles $\theta$ as we display in Fig. 3, which is equivalent to the Meissner response for a fixed nematic angle but with the magnetic field rotated in the $x-y$ plane. The $K_{x x}^{(S)}$ and $K_{y y}^{(S)}$
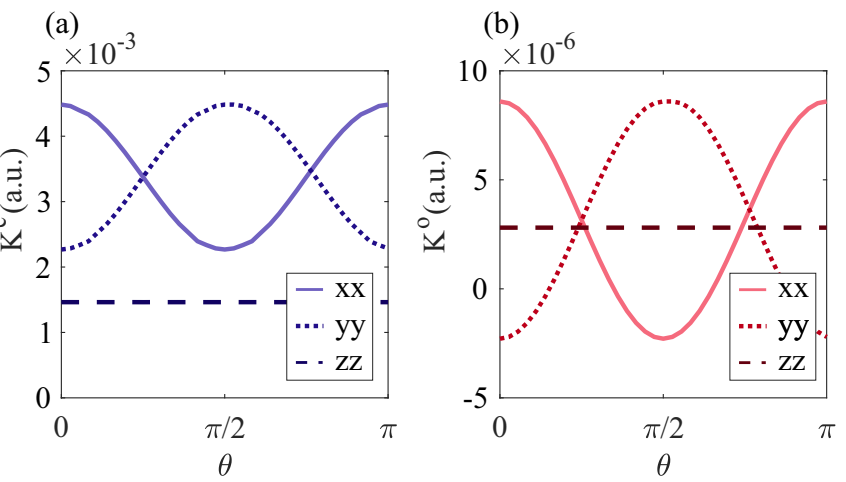

FIG. 3. In- and out-of-plane (a) even- and (b) odd-frequency contributions to the Meissner response as a function of nematic angle $\theta$ for chemical potential $\mu=0.3 \mathrm{eV}$.

responses display a clear twofold periodicity as a function of nematic angle both for the even- and the odd-frequency response, while the out-of-plane $K_{z z}^{(S)}$ response is completely independent of the choice of $\theta$. This twofold periodic response is a clear-cut manifestation of the spontaneous rotational symmetry breaking by the superconducting order parameter, as the Meissner effect explicitly measures the superconducting state. The variation is significant, with $\max \left(K_{x x}^{(S)}\right) / \min \left(K_{x x}^{(S)}\right) \approx 2$, even though the very small order parameter hardly affects the band dispersion.

Before concluding, we note that the response presented in Fig. 3 only contains the superconducting contribution to the Meissner effect. The total Meissner response $K_{\mu \nu}$ requires also including the first term in Eq. (3), as well as the contribution from the diamagnetic current operator for corrections to the low-energy spectrum beyond linear order in k. However, adding any of these neglected terms will only overlay additional two- or sixfold symmetric quantities on top of the very pronounced twofold rotation symmetry in Fig. 3. Thus, we still expect a clear signature of nematic superconductivity in the Meissner effect, experimentally detectable, e.g., in the London penetration depth.

Conclusions. In conclusion, we identify large and dominating odd-frequency intraorbital $s$-wave pair correlations in the nematic superconducting state in doped $\mathrm{Bi}_{2} \mathrm{Se}_{3}$, even though the order parameter itself constitutes only evenfrequency interorbital $s$-wave pairing. With odd-frequency pair correlations known to influence measurable quantities such as Josephson currents, this result is important for understanding the physical behavior of doped $\mathrm{Bi}_{2} \mathrm{Se}_{3}$. We further discover that the odd-frequency intra- and interband contributions to the Meissner response have opposite signs, canceling in an intricate way to give rise to a small, but unexpectedly diamagnetic Meissner response, which stabilizes the superconducting state. This changes the current understanding, where odd-frequency pairing has been assumed to give a paramagnetic Meissner signal, and reveals the possibility to engineer odd-frequency superconductors where enhanced interband contributions give a stable diamagnetic Meissner response. 
Acknowledgments. We are grateful to D. Kuzmanovski for helpful discussions about the Meissner kernel. This work was supported by the Swedish Research Council (Vetenskapsrådet Grant No. 2018-03488), the European Research
Council (ERC) under the European Union's Horizon 2020 research and innovation programme (ERC-2017-StG-757553), the Swedish Foundation for Strategic Research (SSF), and the Knut and Alice Wallenberg Foundation.
[1] H. Zhang, C.-X. Liu, X.-L. Qi, X. Dai, Z. Fang, and S.-C. Zhang, Nat. Phys. 5, 438 (2009).

[2] Y. Xia, D. Qian, D. Hsieh, L. Wray, A. Pal, H. Lin, A. Bansil, D. Grauer, Y. S. Hor, R. J. Cava et al., Nat. Phys. 5, 398 (2009).

[3] Y. S. Hor, A. J. Williams, J. G. Checkelsky, P. Roushan, J. Seo, Q. Xu, H. W. Zandbergen, A. Yazdani, N. P. Ong, and R. J. Cava, Phys. Rev. Lett. 104, 057001 (2010).

[4] L. Fu and E. Berg, Phys. Rev. Lett. 105, 097001 (2010).

[5] K. Matano, M. Kriener, K. Segawa, Y. Ando, and G.-q. Zheng, Nat. Phys. 12, 852 (2016).

[6] S. Yonezawa, K. Tajiri, S. Nakata, Y. Nagai, Z. Wang, K. Segawa, Y. Ando, and Y. Maeno, Nat. Phys. 13, 123 (2016).

[7] Y. Pan, A. M. Nikitin, G. K. Araizi, Y. K. Huang, Y. Matsushita, T. Naka, and A. de Visser, Sci. Rep. 6, 28632 (2016).

[8] T. Asaba, B. J. Lawson, C. Tinsman, L. Chen, P. Corbae, G. Li, Y. Qiu, Y. S. Hor, L. Fu, and L. Li, Phys. Rev. X 7, 011009 (2017).

[9] J. Shen, W.-Y. He, N. F. Q. Yuan, Z. Huang, C.-w. Cho, S. H. Lee, Y. S. Hor, K. T. Law, and R. Lortz, npj Quantum Mater. 2, 59 (2017).

[10] S. Yonezawa, Condens. Matter 4, 2 (2019).

[11] L. Fu, Phys. Rev. B 90, 100509(R) (2014).

[12] A. M. Black-Schaffer and A. V. Balatsky, Phys. Rev. B 88, 104514 (2013).

[13] C. Triola, J. Cayao, and A. M. Black-Schaffer, Ann. Phys. 532, 1900298 (2020).

[14] V. L. Berezinskii, JETP Lett. 20, 287 (1974).

[15] F. S. Bergeret, A. F. Volkov, and K. B. Efetov, Phys. Rev. Lett. 86, 4096 (2001).

[16] F. S. Bergeret, A. F. Volkov, and K. B. Efetov, Rev. Mod. Phys. 77, 1321 (2005).

[17] Y. Tanaka and A. A. Golubov, Phys. Rev. Lett. 98, 037003 (2007).

[18] Y. Tanaka, Y. Tanuma, and A. A. Golubov, Phys. Rev. B 76, 054522 (2007).

[19] A. M. Black-Schaffer and A. V. Balatsky, Phys. Rev. B 86, 144506 (2012).

[20] A. M. Black-Schaffer and A. V. Balatsky, Phys. Rev. B 87, 220506(R) (2013).

[21] J. Linder and J. W. A. Robinson, Nat. Phys. 11, 307 (2015).

[22] J. Linder and A. V. Balatsky, Rev. Mod. Phys. 91, 045005 (2019).

[23] F. Parhizgar and A. M. Black-Schaffer, Phys. Rev. B 90, 184517 (2014).

[24] C. Triola, D. M. Badiane, A. V. Balatsky, and E. Rossi, Phys. Rev. Lett. 116, 257001 (2016).

[25] D. Kuzmanovski and A. M. Black-Schaffer, Phys. Rev. B 96, 174509 (2017).

[26] L. Komendová and A. M. Black-Schaffer, Phys. Rev. Lett. 119, 087001 (2017).
[27] C. Triola and A. M. Black-Schaffer, Phys. Rev. B 97, 064505 (2018).

[28] M. Eschrig, J. Kopu, J. C. Cuevas, and G. Schön, Phys. Rev. Lett. 90, 137003 (2003).

[29] Y. Asano, Y. Tanaka, and A. A. Golubov, Phys. Rev. Lett. 98, 107002 (2007).

[30] F. Parhizgar and A. M. Black-Schaffer, arXiv:1810.09687.

[31] P. Dutta and A. M. Black-Schaffer, Phys. Rev. B 100, 104511 (2019).

[32] Y. Tanaka, Y. Asano, A. A. Golubov, and S. Kashiwaya, Phys. Rev. B 72, 140503(R) (2005).

[33] S. Mironov, A. Mel'nikov, and A. Buzdin, Phys. Rev. Lett. 109, 237002 (2012).

[34] J. A. Ouassou, W. Belzig, and J. Linder, Phys. Rev. Lett. 124, 047001 (2020).

[35] A. Di Bernardo, Z. Salman, X. L. Wang, M. Amado, M. Egilmez, M. G. Flokstra, A. Suter, S. L. Lee, J. H. Zhao, T. Prokscha et al., Phys. Rev. X 5, 041021 (2015).

[36] Y. Asano and A. Sasaki, Phys. Rev. B 92, 224508 (2015).

[37] E. Abrahams, A. Balatsky, D. J. Scalapino, and J. R. Schrieffer, Phys. Rev. B 52, 1271 (1995).

[38] P. Coleman, E. Miranda, and A. Tsvelik, Phys. Rev. B 49, 8955 (1994).

[39] R. Heid, Z. Phys. B 99, 15 (1995).

[40] G. Rosenberg and M. Franz, Phys. Rev. B 85, 195119 (2012).

[41] R. Tao, Y.-J. Yan, X. Liu, Z.-W. Wang, Y. Ando, Q.-H. Wang, T. Zhang, and D.-L. Feng, Phys. Rev. X 8, 041024 (2018).

[42] E. Lahoud, E. Maniv, M. S. Petrushevsky, M. Naamneh, A. Ribak, S. Wiedmann, L. Petaccia, Z. Salman, K. B. Chashka, Y. Dagan et al., Phys. Rev. B 88, 195107 (2013).

[43] See Supplemental Material at http://link.aps.org/supplemental/ 10.1103/PhysRevB.101.180512 for the anomalous Green's function to infinite order in $\Delta$, a detailed derivation of the Meissner kernel, and the explicit Matsubara summation including the splitting into intra- and interband processes, which includes Refs. [44-46].

[44] G. D. Mahan, Many-Particle Physics (Springer, New York, 2000).

[45] D. Kuzmanovski and M. G. Vavilov, Supercond. Sci. Technol. 25, 084001 (2012).

[46] N. B. Kopnin and E. B. Sonin, Phys. Rev. Lett. 100, 246808 (2008).

[47] D. J. Scalapino, S. R. White, and S. C. Zhang, Phys. Rev. Lett. 68, 2830 (1992).

[48] S. Peotta and P. Törmä, Nat. Commun. 6, 8944 (2015).

[49] L. Liang, T. I. Vanhala, S. Peotta, T. Siro, A. Harju, and P. Törmä, Phys. Rev. B 95, 024515 (2017).

[50] T. Mizoguchi and M. Ogata, J. Phys. Soc. Jpn. 84, 084704 (2015). 